Ann. Biol. anim. Bioch. Biophys., I973, 13 (4), 587-609.

\title{
EFFETS DES HUILES DE COLZA ET DE CANBRA (1) SUR LES LIPIDES ET L'ANATOMIE DU MYOCARDE DU RAT : ÉTUDE A COURT TERME (de 0 à 60 jours)
}

\author{
G. ROCQUELIN, J.-P. SERGIEL, P. O. ASTORG et R. CLUZAN* \\ avec la collaboration technique de Lydia Recorbet, P. JUANedA et J.-C. Peleran \\ Station de Recherches sur la Qualité des Aliments de l'Homme, I. N. R. A., \\ 21034 Dijon Cedex \\ *Laboratoire d'Anatomie pathologique, Hôpital Saint-Michel, \\ 33, rue Olivier de Serres, \\ 75015 Paris
}

\section{RÉSUMÉ}

Les auteurs ont étudié les effets de 3 huiles végétales raffinées (arachide, colza, canbra) sur les lipides et l'anatomie du myocarde du Rat. Les régimes expérimentaux contenaient i 5 p. Iоo en poids (30 p. Ioo des calories) de lipides. Les rats, pris au sevrage, ont été sacrifiés après 3,7, I5, 30, 60 jours de mise au régime. L'huile de colza provoque très rapidement dans le myocarde une accumulation importante de triglycérides riches en monoènes à longue châne (acides érucique et gadoléique) qui atteint un maximum au cours de la première semaine. Ce stockage anormal de monoènes à longue chaîne dans les triglycérides cardiaques entraîne celui d'autres acides gras (oléique et linoléique) et une modification de la structure glycéridique. La stéatose cardiaque disparaît peu à peu dans le temps mais après 60 jours d'expérience on retrouve encore des quantités non négligeables d'acide érucique dans l'organe $(8, \mathrm{I} \mathrm{p}$. roo). L'hypothèse d'une adaptation métabolique du myocarde est suggérée. L'acide érucique se retrouve aussi dans les phospholipides cardiaques $\left(4,8\right.$ à $\mathrm{I} 2 \mathrm{P}$. Ioo) dès le $3^{\mathrm{e}}$ jour de mise au régime. L'ensemble de ces phénomènes ne se retrouve pas avec les huiles d'arachide et de canbra.

Les huiles de colza et de canbra, apportant de l'acide linolénique, modifient le spectre des acides polyinsaturés, $\mathrm{C}_{20}$ et $\mathrm{C}_{22}$ en $\omega_{6}$ et $\omega_{3}$, du myocarde.

Des lésions myocardiques ont été à nouveau retrouvées chez les rats ingérant aussi bien de l'huile de colza que de l'huile de canbra. Ces résultats reposent le problème du lien de cause à effet qui peut exister entre la stéatose et la lésion cardiaque et celui de la responsabilité unique de l'acide érucique dans ces deux effets pathologiques.

(1) Canbra = Contraction de Canadian brassica. Nom donné par les généticiens canadiens à l'huile de colza très pauvre en acide érucique. 


\section{INTRODUCTION}

De nombreuses études ont montré que les huiles de colza ou de navette riches en acides érucique et gadoléique provoquent au niveau du myocarde de plusieurs espèces animales une accumulation précoce et importante de triglycérides riches en monoènes à longue chaîne $\left(\mathrm{C}_{20}\right.$ et $\left.\mathrm{C}_{22}\right)$. On trouvera sur ce sujet, deux mises au point bibliographiques récentes (Rocquelin et CluZAN, I97I ; AbDELLATIF, I972).

Chez le Rat, la stéatose cardiaque diminue peu à peu dans le temps même si on maintient l'animal à un régime contenant de 1'huile de colza (VLEs et ABDELLATIF, I970; ABDELLATIF et VlES, I970 $a$; ABDELlatiff et VlES, I973; BEAREROGERs et al., I97I). Par contre, des lésions myocardiques apparaissent, qui ont été décrites par ailleurs (ROCQUEIIN et CLUZAN, I968; BEARE-RogERS et NERA, I972 $a$, ABDELLATIF et VLES, I970 $b$, ABDELLATIF et Vles, I97I ; Vodovar et al., I972; BODAK, I972).

L'huile de canbra très pauvre en acide érucique $(0,5 \mathrm{p}$. Ioo à $2 \mathrm{p}$. Ioo des acides gras totaux), ne cause pas à très court terme ( 3 jours de mise au régime) d'accumulation de lipides dans le myocarde du Rat mais provoque néanmoins des lésions anatomiques de l'organe (ROCQUELIN et CLUZAN, I968 ; RoCQUELIN, I972).

Dans l'étude présente, nous avons eu pour double but :

- de chercher par des méthodes biochimiques et histologiques si l'huile de canbra n'entraînerait pas une modification des lipides cardiaques plus tardive que celle obtenue avec l'huile de colza : s'il en était ainsi, un argument de poids serait apporté en faveur de 1'existence d'un lien de cause à effet entre stéatose et myocardite ;

- de compléter les connaissances, d'une part sur l'influence dans le temps des huiles de colza et de canbra sur les lipides cardiaques du Rat (aspects qualitatif et quantitatif), et, d'autre part, sur la nature et l'évolution des lésions de l'organe.

Du fait de l'intérêt suscité dans de nombreux laboratoires par les effets physiopathologiques des huiles de crucifères, plusieurs recherches sont actuellement effectuées sur ce sujet. C'est ainsi que quelques études récentes de l'influence des huiles de colza sur les lipides du cour et d'autres organes (WALKER et al., I972; WALKER, I972) ont déjà paru, études que le présent travail complète ou confirme. Par contre, les travaux sur l'huile de canbra, dans ce domaine, sont beaucoup plus rares.

\section{MATÉRIELS ET MÉTHODES}

\section{A. - Conduite des expériences sur animaux}

Cent soixante cinq rats Wistar mâles pris à 1'âge de 3 semaines sont disposés en cages individuelles et nourris pendant 3 jours avec un régime d'élevage commercial (provende Extralabo) dépourvu de monoènes à très longue chaîne $\left(\mathrm{C}_{20}: 1, \mathrm{C}_{22}: 1\right.$ ). Ils sont alors répartis en 3 lots de 55 animaux qui reçoivent des régimes expérimentaux ne différant les uns des autres que par la nature des lipides. Ces régimes ont la composition suivante (en $\mathrm{g}$ pour Ioo $\mathrm{g}$ de matière sèche) : caséine lactique : 18 - amidon de froment : 37 - saccharose : 24 - lipides : 15 - agar-agar : 2 - mélange salin complet de HuBBEL et al. : 4. Un mélange vitaminique adéquat (1) est ajouté au régime, qui

(1) On trouvera sa composition dans une publication précédente (Rocouelin et Cluzan, I968). 
est distribué sous forme de bouillie semi-liquide (2/3 de matière sèche $-\mathrm{I} / 3$ d'eau distillée). Trois huiles raffinées ont été étudiées : une huile d'arachide (prise comme produit témoin), une huile de colza riche en acide érucique, une huile de canbra très pauvre en acide érucique. La composition en acides gras des huiles est donnée par le tableau I. On remarquera le taux très faible d'acide érucique $(0,4 \mathrm{p}$. IOo) de l'huile de canbra.

TABLEAU I

Composition (p. ıоo) en acides gras des huiles végétales étudiées

\begin{tabular}{|c|c|c|c|}
\hline Acides gras identifiés & Arachide & Colza & Canbra \\
\hline 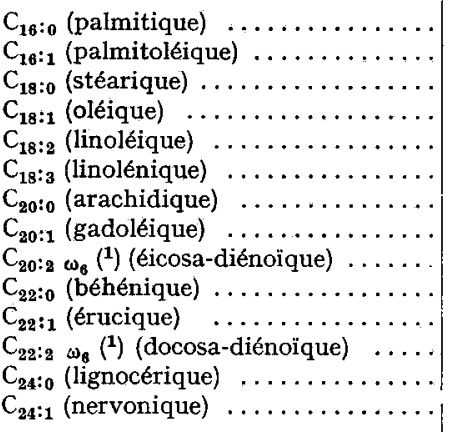 & $\begin{array}{r}10,5 \\
0,3 \\
3,0 \\
49,3 \\
29,2 \\
\overline{1,4} \\
1,3 \\
\overline{2,7} \\
\overline{0,4} \\
\overline{1,3} \\
0,5\end{array}$ & $\begin{array}{r}3,4 \\
0,1 \\
0,9 \\
11,4 \\
14,4 \\
7,9 \\
0,8 \\
7,1 \\
0,6 \\
0,6 \\
50,6 \\
0,9 \\
\overline{1,3}\end{array}$ & $\begin{array}{r}4,7 \\
0,4 \\
2,4 \\
59,8 \\
21,3 \\
7,2 \\
1,0 \\
1,5 \\
0,2 \\
0,3 \\
0,4 \\
- \\
- \\
0,9\end{array}$ \\
\hline
\end{tabular}

(1) Identification supposée.

Les animaux sont nourris chaque jour ad libitum (régime et eau de boisson). Ils sont sacrifiés par décapitation, après un jeùne de 5 à 6 heures, les $3^{e}, 7^{\circ}, 15^{\circ}, 30^{\circ}$ et $60^{\circ}$ jours de mise au régime. A chaque stade, 3 à 5 rats par lot sont utilisés pour les analyses de jipides d'organes et 5 à I 6 rats pour les études anatomiques. Le sang de l'animal est prélevé par ponction cardiaque sous légère anesthésie à l'aide d'une seringue héparinée. Le plasma est ensuite obtenu par centrifugation du sang. Aussitôt après le sacrifice, le coeur est enlevé, pesé puis conservé soit dans un mélange de chloroforme-méthanol $(2 / \mathrm{I}-\mathrm{V} / \mathrm{V})$ à - I $5^{\circ} \mathrm{C}$ pour les études biochimiques des lipides, soit dans les liquides de fixation pour les observations histologiques.

\section{B. - Extraction et analyse des lipides d'organes}

Les lipides sont extraits par la méthode de Folch et al. (I957). L'extrait lipidique total est đébarrassé de son solvant par évaporation sous vide à $40^{\circ} \mathrm{C}$ dans un évaporateur rotatif, séché à l'alcool absolu puis pesé. Il est repris dans du chloroforme-méthanol (2/I-V/V) et une partie aliquote (r/ro à $\mathrm{I} / 20$ de l'extrait total) est prélevée pour l'analyse des acides gras totaux. La séparation et le dosage quantitatif des classes de lipides cardiaques sont effectués, à partir de pools de 3 à 5 organes, par chromatographie sur couches minces préparatives de silicagel d'une épaisseur de $500 \mu$. Le gel de silice est préalablement lavé au chloroforme-méthanol $(2 / \mathrm{I}-\mathrm{V} / \mathrm{V})$, séché et activé pendant 2 heures à I I $0^{\circ} \mathrm{C}$. Io à $30 \mathrm{mg}$ de lipides cardiaques sont déposés par plaques de $20 \times 20 \mathrm{~cm}$ à l'aide d'un applicateur d'échantillon. Le mélange de solvants utilisé pour séparer les lipides est le suivant : hexane-éther éthylique-méthanol-acide acétique, 90/20/3/2 selon BRown et Johnston (I962). Les lipides ainsi séparés sont identifiés par révélation des bandes en pulvérisant sur les plaques une solution à 0,2 p. Ioo de $2^{\prime}-7^{\prime}$ dichlorofluorescéine dans l'éthanol ab solu et comparaison des Rf à ceux d'étalons connus. Les lipides sont ensuite récupérés par grattage des bandes de gel de silice qui sont directement transvasées dans des tubes de Io ml à fermeture étanche (joint en téflon). On ajoute alors une quantité connue de standard interne (acide heptadécanoïque) et les lipides sont transméthylés à $95^{\circ} \mathrm{C}$ pendant 3 heures dans 5 à Io ml d'un mélange 
benzène-méthanol-acide sulfurique concentré, 5/10/1-V/V/V selon Munsch, (I970). Les esters méthyliques des acides gras transvasés dans une ampoule à décanter, sont extraits deux fois avec de l'hexane après addition de $4^{\circ} \mathrm{ml}$ d'eau. Tous les solvants utilisés sont rigoureusement purs ou redistillés au laboratoire avant usage.

Les esters méthyliques sont séparés, identifiés et dosés par chromatographie en phase gazeuse. Nous employons des colonnes en acier inox de $3 \mathrm{~m}$ de longueur et d'un diamètre extérieur de $3 \mathrm{~mm}$, remplies au laboratoire avec des phases stationnaires polaires (butanediol succinate, BDS, ou diéthylène glycol succinate, DEGS, 8 à Io p. Ioo en poids) sur chromosorb W-AW-HMDS 6o/ 80 mesh. Elles sont utilisées en isotherme à $190^{\circ} \mathrm{C}$ (pour les colonnes de BDS) ou $\mathrm{I}^{\circ} 0^{\circ} \mathrm{C}$ (pour les colonnes de DEGS). Nos appareils sont équipés de détecteurs à ionisation de flamme d'hydrogène. Les esters méthyliques sont identifiés par comparaison de leurs distances de rétention avec celles d'échantillons biologiques connus ou de standards du commerce ( ${ }^{1}$ ) analysés dans les mêmes conditions. Les aires sous les pics sont calculées en effectuant le produit de la hauteur du pic et de la largeur à mi-hauteur.

\section{C. - Détermination de la distribution des acides gras sur les molécules de glycérol dans les triglycérides cardiaques}

Les triglycérides cardiaques, isolés par chromatographie sur couches minces préparatives, sont soumis à une hydrolyse lipasique selon la méthode de Ludoy et al. (1964), qui permet de travailler sur de faibles quantités de produit (10 à $20 \mathrm{mg}$ dans le cas présent). Les 2-monoglycérides formés au cours de la lipolyse sont séparés des autres glycérides par chromatographie sur couches minces puis transméthylés selon les techniques décrites dans le paragraphe précédent. L'analyse des acides gras totaux constitutifs des triglycérides et des 2-monoglycérides est faite par chromatographie en phase gazeuse et la proportion de chaque acide gras estérifié sur la position centrale des glycérides est calculée selon la formule de MATTson et Volpenhein (I96I).

\section{D. - Techniques histologiques}

Les viscères prélevés aux différents moments de sacrifice ont été fixés :

- dans le liquide de Bouin pour étude histologique en paraffine ;

- en partie dans le formol-calcium de Baker, suivi d'une post-chromisation pour étude des lipides.

Le cour, en particulier, a été sectionné longitudinalement selon son grand axe et selon un plan frontal avec fixation d'une des parties dans le liquide de Bouin, et l'autre dans le formol-calcium.

Cette technique avait un double but :

- d'une part préciser la topographie éventuelle de la surcharge lipidique,

-_ d'autre part, d'étudier la topographie des lésions selon une autre technique (les précédentes études ayant été conduites selon des coupes transversales, perpendiculaires au grand axe du coeur).

Les colorations utilisées ont été :

- pour les coupes en paraffine : hématéine - éosine, trichrome de Masson, orcéine, réticuline, selon Gordon-Sweet.

- pour les coupes en congélation (après fixation au formol-calcium) : oil red O, Soudan III, Soudan IV, sulfate de bleu de Nils.

\section{RÉSULTATS}

\section{I. - Poids du cour (tabl. 2)}

Comme dans les études précédentes, on retrouve une augmentation du poids du cœur chez les rats ingérant de l'huile de colza. Cette augmentation est surtout marquée aux $7^{\mathrm{e}}$ et $\mathbf{I}_{5}{ }^{\mathrm{e}}$ jours d'expérience.

(1) Mélanges Supelco, Inc. P. U. F. A. no I comprenant: $\mathrm{C}_{16}: 0, \mathrm{C}_{18}: 0, \mathrm{C}_{20: 0}, \mathrm{C}_{18}: 1, \mathrm{C}_{20: 1}, \mathrm{C}_{22: 1}$,

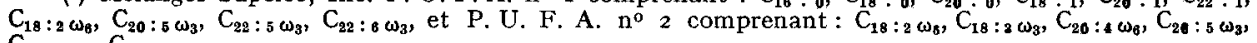

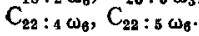




\section{2. - Teneur en lipides totaux du myocarde (fig. I)}

La teneur en lipides totaux du myocarde des rats ingérant de l'huile de colza est beaucoup plus élevée que celle des autres lots et atteint un maximum au $7^{\mathrm{e}}$ jour de mise au régime. Elle diminue ensuite mais ne redevient comparable à celle du lot témoin (arachide) que vers le $60^{\mathrm{e}}$ jour. On remarque que l'huile de canbra ne provoque pas d'accumulation anormale de lipides dans l'organe à aucun moment de l'expérience. Les études histologiques (voir $\S 7$ ) confirment ce résultat. Les effets de l'huile de canbra sont, à cet égard, identiques à ceux de l'huile d'arachide.

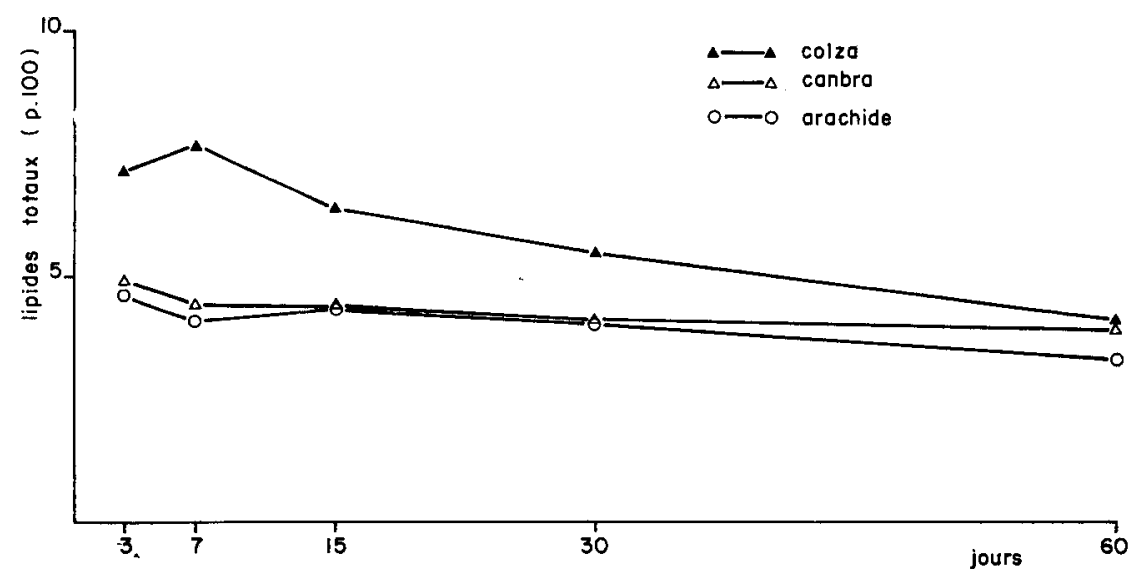

FIG. r. - Infuence à court terme des huiles du rêgime sur les lipides totaux du myocarde (moyennes établies sur 3 rats par lot et par stade de sacrifice)

La dispersion des valeurs obtenues (minimum et maximum) est la suivante :

\begin{tabular}{|c|c|c|c|c|c|c|c|c|c|c|}
\hline & \multicolumn{2}{|c|}{3 jours } & \multicolumn{2}{|c|}{7 jours } & \multicolumn{2}{|c|}{15 jours } & \multicolumn{2}{|c|}{30 jours } & \multicolumn{2}{|c|}{60 jours } \\
\hline & $\min$. & $\max$. & $\min$. & $\max$. & $\min$. & $\max$ & $\min$. & $\max$. & $\min$. & $\max$ \\
\hline Arachide ..... & 4,5 & 4,7 & 3,0 & 5,0 & 4,0 & 4,8 & 3,7 & 4,3 & 3,3 & 3,4 \\
\hline Colza ....... & 6,0 & 8,1 & 5,4 & 9,1 & 5,0 & 8,1 & 4,9 & 6,7 & 3,7 & 5,0 \\
\hline Canbra...... & 4,4 & 5,3 & 4,1 & 4,8 & 4,1 & 4,7 & 3,8 & 4,8 & 3,5 & 4,7 \\
\hline
\end{tabular}

\section{3. - Triglycérides et phospholipides cardiaques (fig. 2)}

Les proportions relatives des triglycérides et des phospholipides obtenues dans les myocardes des rats témoins (arachide) montrent que les phospholipides cardiaques sont quantitativement beaucoup plus abondants que les triglycérides, ce qui pæut être considéré comme normal. On constate néanmoins ici, chez les témoins, une légère augmentation de la proportion de triglycérides du $7^{\mathrm{e}}$ au $30^{\mathrm{e}}$ jour d'expérience. L'huile de canbra ne semble pas entraîner de différence marquée par rapport à 1'huile 


\begin{tabular}{|c|c|c|c|c|c|}
\hline & \multicolumn{5}{|c|}{ Arachide } \\
\hline Durée de l'expérience (jours) & 3 & 7 & 15 & 30 & 60 \\
\hline Nombre de rats par sacrifice & 8 & 9 & 10 & 9 & 20 \\
\hline Poids du myocarde ( $\left.{ }^{\mathbf{l}}\right)$ & $\begin{array}{r}0,442 \\
\pm 0,017\end{array}$ & $\begin{array}{r}0,503 \\
\pm 0,011\end{array}$ & $\begin{array}{r}0,619 \\
\pm 0,023\end{array}$ & $\begin{array}{r}0,916 \\
\pm 0,034\end{array}$ & $\begin{array}{r}1,197 \\
\pm 0,040\end{array}$ \\
\hline $\begin{array}{l}\text { Poids du myocarde }(1) \\
\text { p. } 100 \mathrm{~g} \text { de poids vif }\end{array}$ & $\begin{array}{r}0,541 \\
\pm 0,020\end{array}$ & $\begin{array}{r}0,520 \\
\pm 0,015\end{array}$ & $\begin{array}{r}0,455 \\
\pm 0,012\end{array}$ & $\begin{array}{r}0,357 \\
\pm \quad 0,010\end{array}$ & $\begin{array}{r}0,324 \\
\pm 0,015\end{array}$ \\
\hline
\end{tabular}

(1) Poids exprimé en $\mathrm{g} \pm$ erreur standard de la moyenne.

(2) Différence significative au seuil de $P=0,05$ (par rapport au lot Arachide).

${ }^{3}$ ) Différence significative au seuil de $\mathrm{P}=0,01$ (par rapport au lot Arachide).

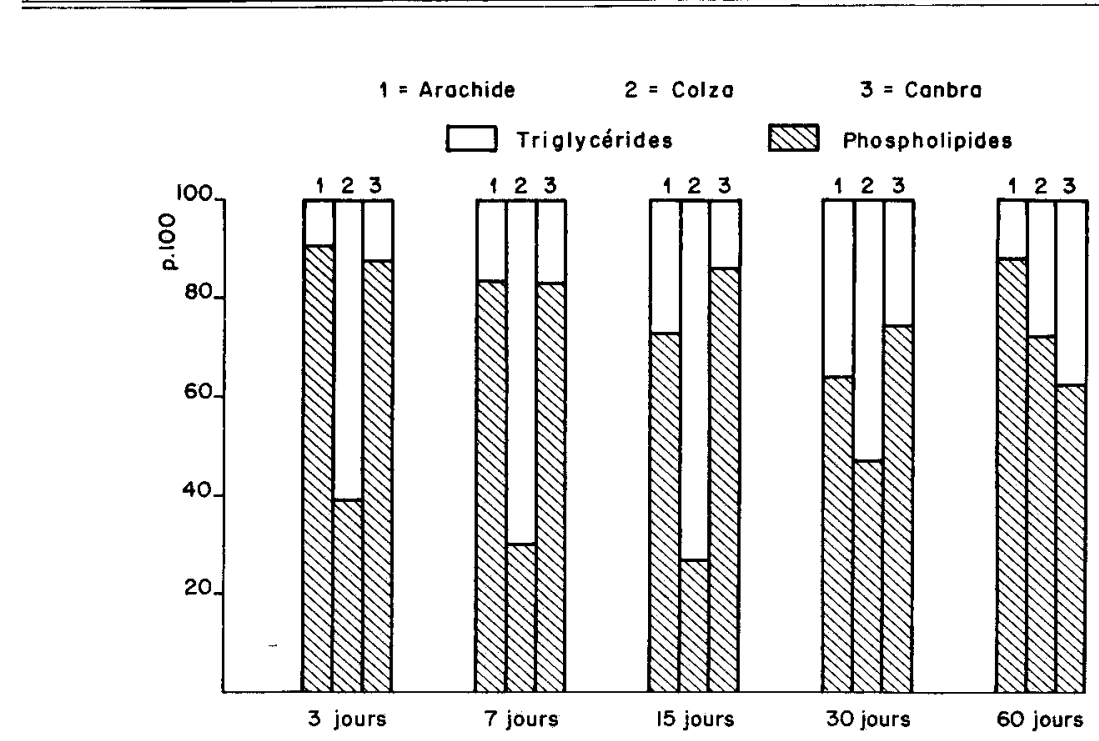

FIG. 2. - Proportions relatives des triglycérides et des phospholipides cardiaques Influence des huiles du régime et de la durée de l'expérience

Résultats obtenus sur pools de lipides de 3 à 4 myocardes par lot et par stade de sacrifice

d'arachide sauf, vers la fin de l'expérience, où l'on constate aussi une augmentation de la proportion des triglycérides. Ces déterminations ayant été faites sur pools d'organes, il n'est pas possible ici de dire si ces variations ont une réelle signification. Enfin, avec 1'huile de colza, on observe des résultats nettement différents des précé- 
Huile de régime

\begin{tabular}{|c|c|c|c|c|c|c|c|c|c|}
\hline \multicolumn{5}{|c|}{ Colza } & \multicolumn{5}{|c|}{ Canbra } \\
\hline 3 & 7 & 15 & 30 & 60 & 3 & 7 & 15 & 30 & 60 \\
\hline 8 & 8 & 10 & 9 & 15 & 8 & 10 & 10 & 11 & 19 \\
\hline $\begin{array}{r}0,427 \\
+0,018\end{array}$ & $\begin{aligned} & 0,538\left(^{(2)}\right. \\
\pm & 0,011\end{aligned}$ & $\begin{aligned} & 0,678\left(^{(3)}\left({ }^{(5)}\right)\right. \\
\pm & 0,027\end{aligned}$ & $\begin{array}{r}0,953 \\
\pm 0,034\end{array}$ & $\begin{array}{r}1,299\left(^{(4)}\right. \\
\pm 0,037\end{array}$ & $\begin{array}{r}0,412 \\
\pm 0,011\end{array}$ & $\begin{array}{r}0,510 \\
\pm 0,016\end{array}$ & $\begin{array}{r}0,546 \\
\pm 0,040\end{array}$ & $\begin{array}{r}0,849 \\
\pm 0,036\end{array}$ & $\begin{array}{r}1,155 \\
\pm 0,028\end{array}$ \\
\hline $\begin{array}{r}0,541 \\
+0,020\end{array}$ & $\begin{array}{r}0,550\left({ }^{4}\right) \\
\pm 0,015\end{array}$ & $\begin{aligned} & 0,550\left(^{(3)()^{(4)}}\right. \\
\pm & 0,011\end{aligned}$ & $\begin{array}{r}0,399 \\
\pm 0,020\end{array}$ & $\begin{array}{r}0,350{ }^{(5)} \\
\pm 0,009\end{array}$ & $\begin{array}{r}0,522 \\
\pm 0,012\end{array}$ & $\begin{array}{r}0,495 \\
\pm 0,011\end{array}$ & $\begin{array}{r}0,468 \\
\pm 0,028\end{array}$ & $\begin{array}{r}0,366 \\
\pm 0,008\end{array}$ & $\begin{array}{r}0,309 \\
\pm 0,006\end{array}$ \\
\hline
\end{tabular}

(4) Différence significative au seuil de $\mathrm{P}=0,05$ (par rapport au lot Canbra).

${ }^{(5)}$ Différence significative au seuil de $\mathrm{P}=0,01$ (par rapport au lot Canbra).

dents depuis le $3^{\mathrm{e}}$ jour jusqu'au $30^{\mathrm{e}}$ jour d'expérience. Le rapport $\frac{\text { triglycérides }}{\text { phospholipides }}$ est supérieur à I et varie de I,I2 à 2,68 (chez les témoins ce rapport varie de $0, I 4$ à $0,56)$. On note, au $I_{5}{ }^{\mathrm{e}}$ jour d'expérience, une valeur de ce rapport légèrement supérieure à celle obtenue au $7^{\mathbf{e}}$ jour bien que la teneur en lipides totaux du myocarde soit maximum au $7^{\mathrm{e}}$ jour (voir fig. I). Ceci peut en partie s'expliquer par la dispersion relativement grande des résultats obtenus à ces stades de l'expérience et le nombre peu élevé d'animaux que nous utilisons à chaque sacrifice. Un rat sur 3 au I $5^{\mathrm{e}}$ jour présentait encore une infiltration graisseuse du myocarde très marquée, plus importante $q u^{\prime}$ au $7^{\circ}$ jour. Ces variations individuelles ne changent pas en fait l'interprétation du phénomène dans son ensemble : l'augmentation des lipides totaux du myocarde provoquée par l'huile de colza est essentiellement due à une accumulation de triglycérides.

\section{4. - Acides gras des lipides totaux du myocarde (aspects quantitatif et qualitatif)}

a) Évolution des pourcentages des principaux acides gras du myocarde (tabl. 3)

Acides saturés (palmitique et stéarique).

Chez les animaux ingérant de l'huile de colza, et par rapport aux animaux des deux autres lots, on constate que le pourcentage des acides palmitique et stéarique est plus faible au moment où la stéatose cardiaque est la plus prononcée ( $3^{\mathrm{e}}$ et $7^{\mathrm{e}}$ jour). On remarque aussi, pendant cette période de temps, une chute du pourcentage de ces acides gras chez les animaux témoins et chez ceux recevant de l'huile de colza. Cette chute ne se produit pas dans le cas de l'huile de canbra. 


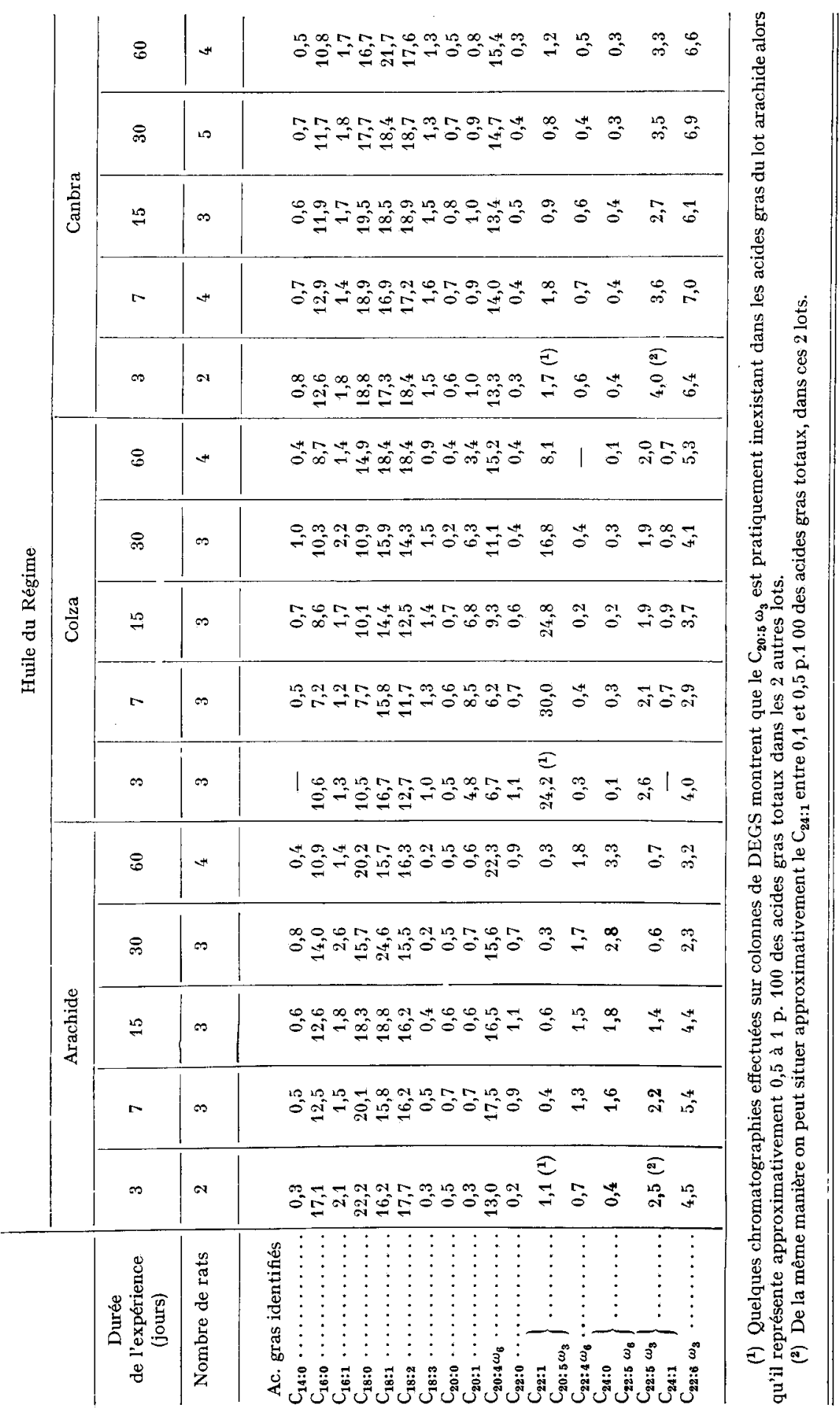


Acides monoinsaturés (oléique, gadoléique, érucique, nervonique).

Outre l'incorporation nette des acides gadoléique et érucique dans le myocarde des rats ayant ingéré de 1'huile de colza, on note que le pourcentage d'acide oléique varie peu en fonction du temps ou de la nature des lipides alimentaires. Cependant, on doit signaler une augmentation de la teneur en acide oléique, an $3^{\circ}$ jour, chez les rats ingérant de 1'huile d'arachide. On verra plus loin ce que cela signifie, en fait, si on exprime ces résultats non plus en pourcentages d'acides gras mais en milligrammes d'acides gras par gramme d'organe.

En ce qui concerne l'apparition d'acide nervonique dans le myocarde des rats du lot " colza ", il est difficile, à ce niveau de l'analyse, de dire si elle a une valeur réelle. Quelques précisions, sur ce point, seront apportées lors de l'étude des lipides en classes (voir $\S 5 b$ ).

\section{Acides polyinsaturés.}

Les pourcentages des acides linolénique et arachidonique sont abaissés dans le myocarde des animaux du lot "colza ", par rapport aux deux autres lots aux $3^{\mathrm{e}}$, $7^{\mathrm{e}}$ et $15^{\mathrm{e}}$ jours d'expérience. Là également, l'interprétation des résultats sera sensiblement modifiée lorsque l'on passera aux masses d'acides gras rapportées par gramme d'organe.

L'apport d'acide linoléique par les huiles de colza et de canbra se traduit au niveau du myocarde par une augmentation du pourcentage de cet acide, par rapport au lot témoin, et par la présence en quantités accrues de ses métabolites supérieurs

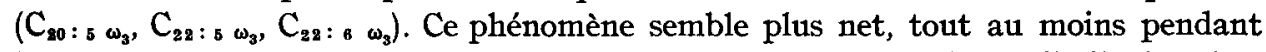
les 5 premiers jours de l'expérience, avec l'huile de canbra qu'avec l'huile de colza.

b) Evolution pondérale des principaux acides gras du myocarde.

On a vu que l'accumulation de lipides constatée dans le myocarde des rats ingérant de l'huile de colza est due essentiellement à une augmentation des triglycérides. Dans ces conditions, la comparaison, entre lots, des pourcentages d'un acide gras donné par rapport aux acides gras totaux de l'organe peut donner des indications insuffisantes voire erronées. Nous avons donc également exprimé nos résultats en milligrammes d'acides gras (sous forme d'esters méthyliques) par gramme d'organe frais.

- Cas des acides érucique $\left(C_{22: 1}\right)$ et gadoléique $\left(C_{20: 1}\right)$ apportés par le régime contenant de l'huile de colza.

La figure 3 montre que les teneurs en ces 2 acides gras augmentent jusqu' au $7^{\mathrm{e}}$ jour (moment où l'accumulation de lipides dans l'organe est maximum) puis diminuent. Néanmoins, si la teneur en lipides totaux du myocarde redevient quasi normale vers le $60^{\circ}$ jour d'expérience (voir fig. I), la présence de ces 2 monoènes à longue chaîne est encore notable à ce moment-là. Ce phénomène ne se retrouve pas dans les 2 autres lots (arachide et canbra).

- Cas des autres acides gras (fig. 4).

Par souci de clarification, nous n'avons reproduit sur cette figure, ni l'évolution pondérale des acides érucique et gadoléique qui a été décrite ci-dessus et sur la figure 3 , ni celle des acides gras quantitativement mineurs (acides polyinsaturés en $\mathrm{C}_{\mathbf{2}}$ par exemple).

On constate pour l'ensemble des lots, une chute des teneurs en acides palmitique (de 24 à $37 \mathrm{p}$. 100) et stéarique (de 16 à $27 \mathrm{p}$. 100) entre le $3^{\mathrm{e}}$ et le $7^{\mathrm{e}}$ jour 
d'expérience. De plus on relève dans le myocarde des rats " colza " une teneur en acide palmitique supérieure à celle des 2 autres lots entre le $3^{\mathrm{e}}$ et le $15^{\mathrm{e}}$ jour d'expérience. Les variations de ces 2 acides gras, au-delà du $15{ }^{e}$ jour, sont, semble-t-il, plus difficiles à analyser et à comparer. Il apparaît, néanmoins, une plus grande stabilité des teneurs en acides palmitique et stéarique dans le lot "canbra ".

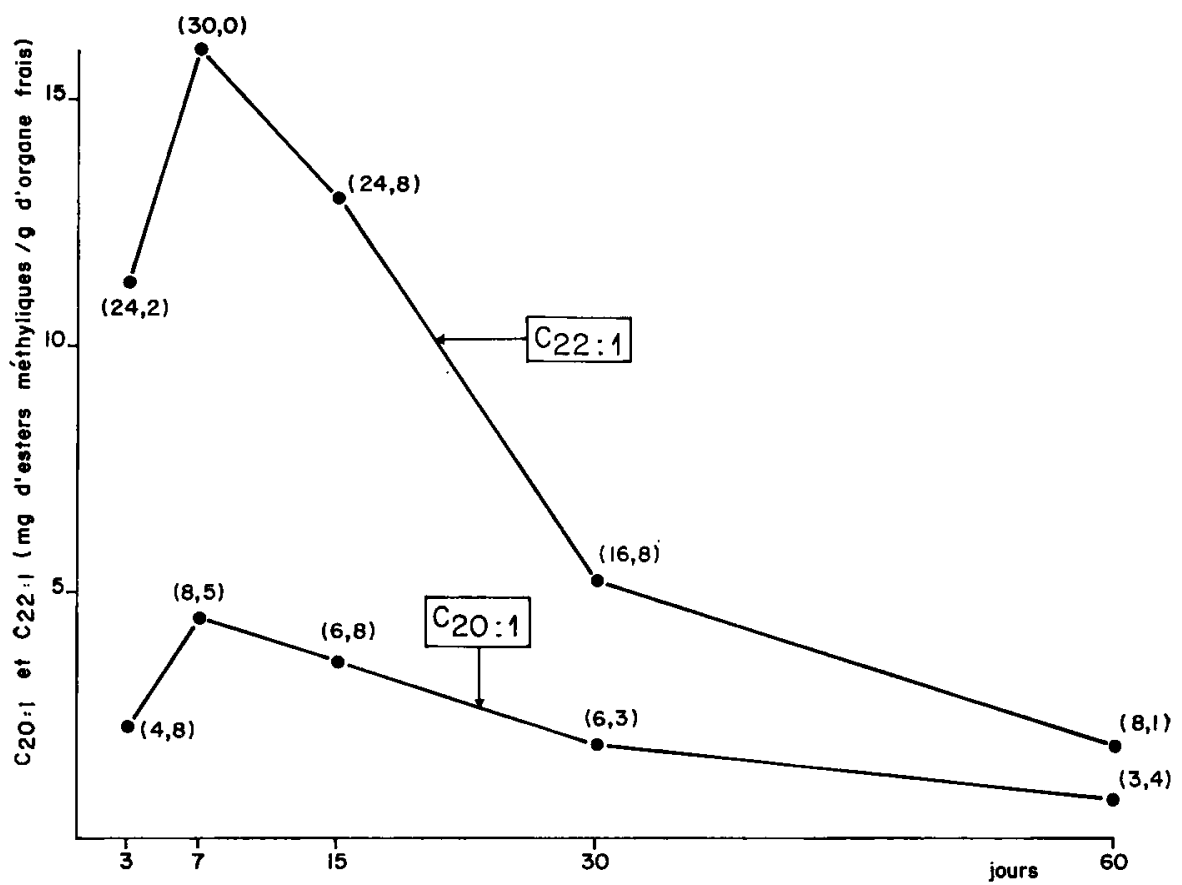

FIG. 3. - Evolution de la teneur en acides érucique $\left(\mathrm{C}_{22: 1}\right)$ et gadoléique $\left(\mathrm{C}_{20: 1}\right)$ du myocarde du Rat ayant ingếé de l'huile de colza depuis l'âge de 24 jours et pendant 60 jours Les nombres entre parenthèses indiquent, pour ces mêmes acides gras, les pourcentages par rapport aux acides gras totaux.

Moyennes établies sur 3 à 4 myocardes par stade de sacrifice

On retrouve également une chute des concentrations en acides oléique et linoléique entre les $3^{\mathrm{e}}$ et $7^{\mathrm{e}}$ jours d'expérience chez les animaux ingérant soit de l'huile d'arachide soit de l'huile de canbra. Il y a ensuite dans le lot " arachide ", une remontée assez nette de l'acide oléique qui passe par un maximum au $3^{\circ}$ jour. Ce résultat est à rapprocher de ce que nous avions observé au niveau des triglycérides à ce même stade (voir fig. 2). Chez les rats du lot " canbra " et au-delà du $7^{\mathrm{e}}$ jour il y a peu de variations.

Dans le cas du lot " colza ", les résultats sont nettement différents. Il y a accumulation prononcée d'acides oléique et linoléique jusqu'aux $7^{\mathrm{e}}$ et $\mathrm{I}^{\mathrm{e}}$ jours d'expérience puis diminution, ces deux phénomènes suivant étroitement la courbe que l'on observe avec les acides érucique et gadoléique (voir fig. 3). Les autres acides gras constitutifs du myocarde et qui ne sont pas représentés sur cette figure, ne suivent pas une telle évolution.

En ce qui concerne l'acide arachidonique, les variations, entre les 3 lots et dans le temps, sont plus atténuées. 

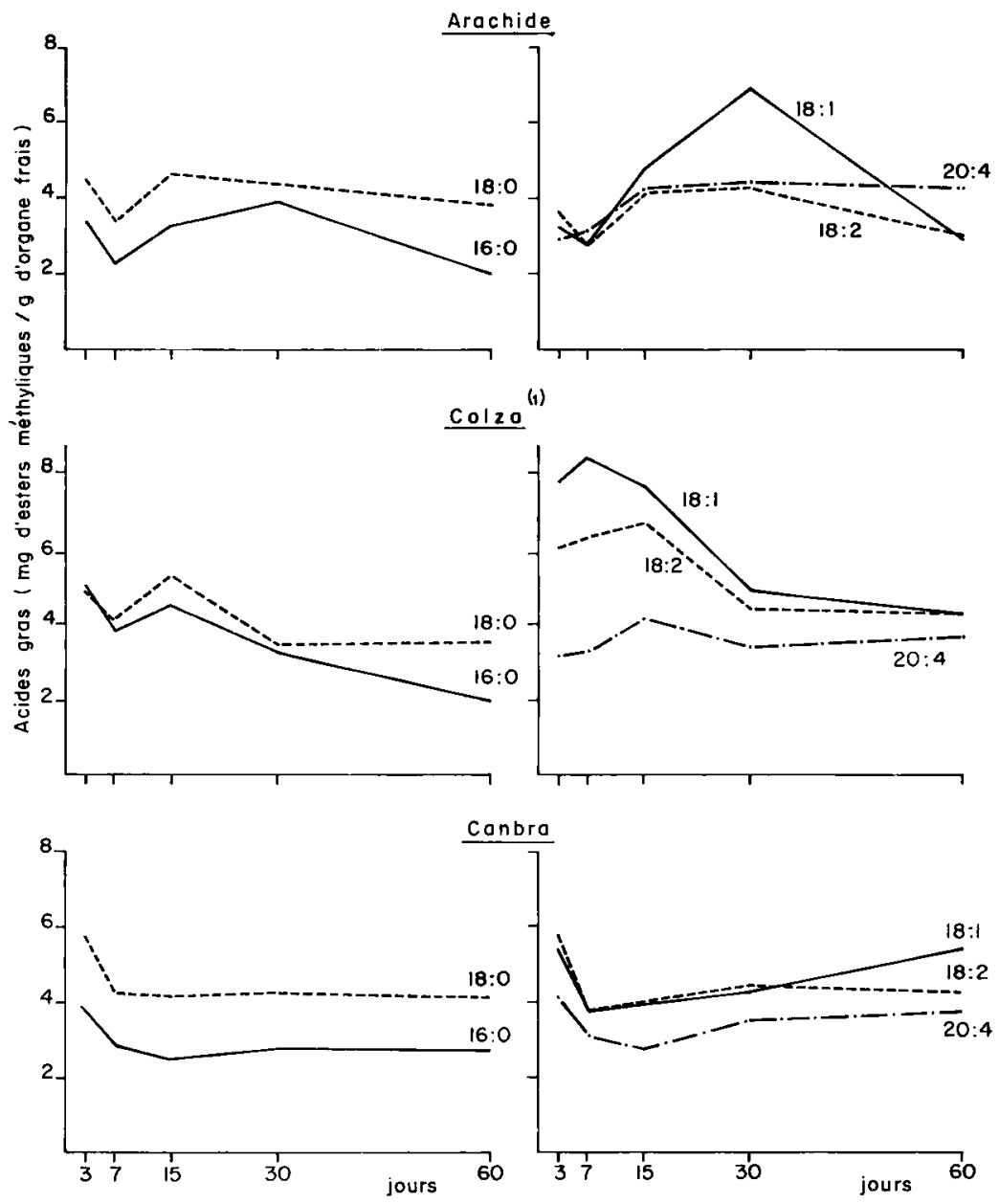

FIG. 4. - Évolution de la tensur (mg/g) des principaux acides gras du myocarde en fonction de la nature des lipides du régime et de la durée de l'experience

(r) En ce qui concerne les acides érucique $\left(C_{22: 1}\right)$ et gadoléique $\left(C_{20: 1}\right)$, voir fig. 3

\section{5. - Acides gras des triglycérides et des phospholipides cardiaques}

a) Cas des triglycérides (tabl. 4).

L'incorporation des acides érucique et gadoléique apportés par l'huile de colza, est très importante dans les triglycérides cardiaques. Ceux-ci représentent jusqu'à 5o p. Ioo des acides gras totaux. Cette incorporation se fait principalement aux dépens des acides palmitique, stéarique et oléique. Au $60^{\circ}$ jour d'expérience, lorsque la teneur en lipides totaux et le rapport $\frac{\text { triglycétides }}{\text { phospholipides }}$ sont redevenus proches de la normale (fig. I et 2), on note encore la présence non négligeable d'acides érucique et gadoléique. 


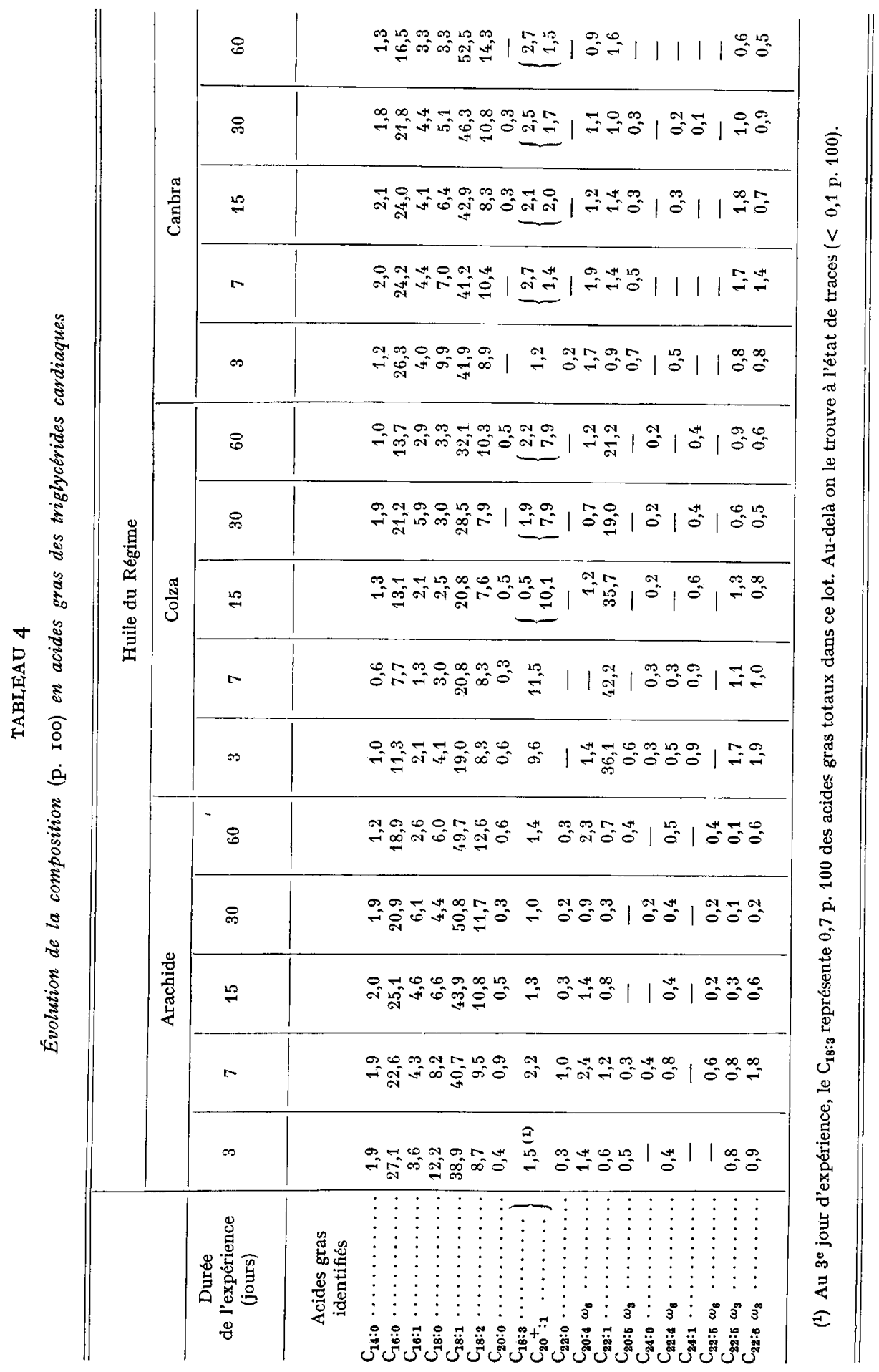




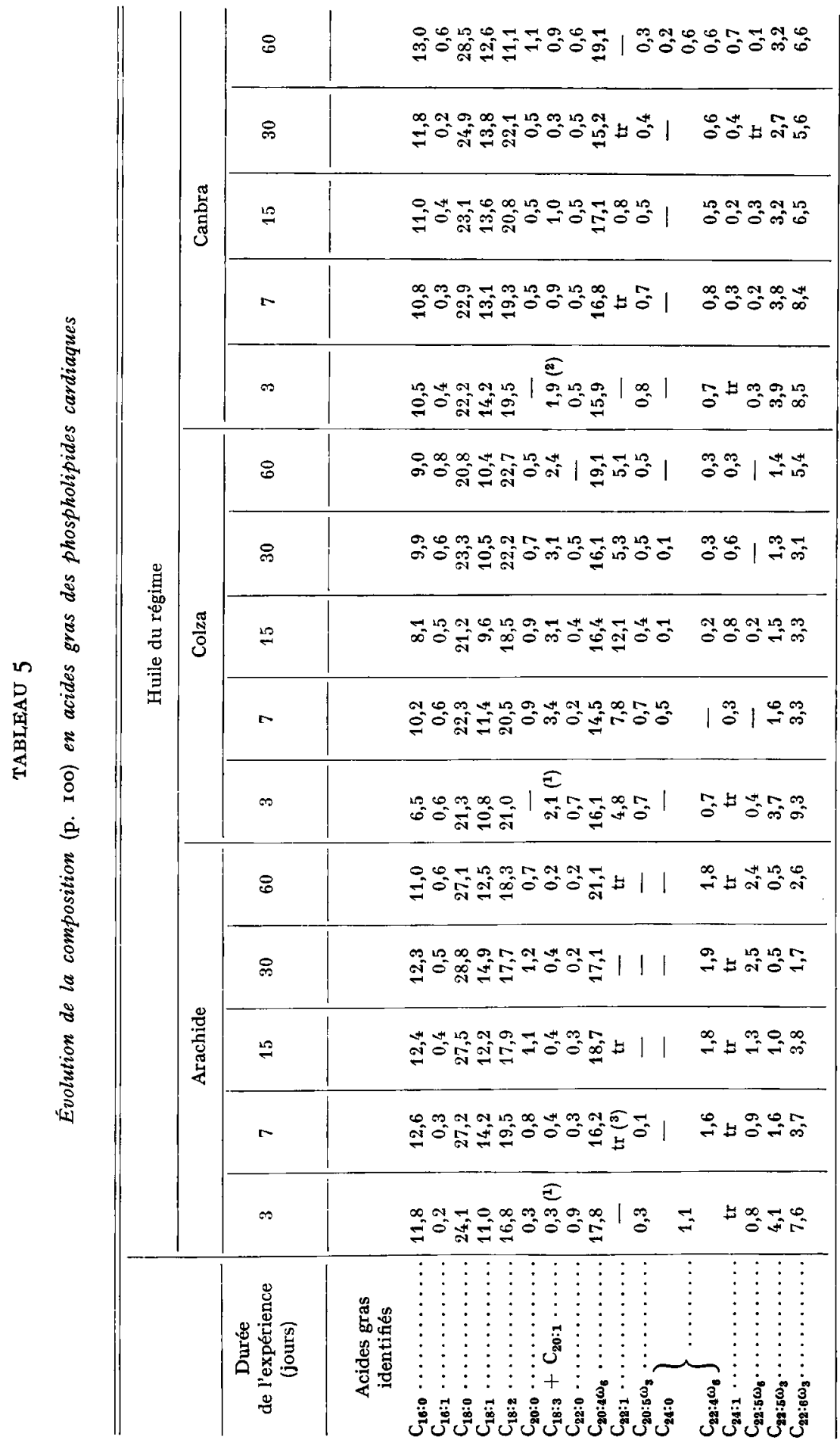


b) Cas des phospholipides (tab1. 5).

Il est intéressant de constater que l'incorporation des acides érucique et gadoléique dans les phospholipides cardiaques des animaux ingérant de l'huile de colza est moins importante que dans les triglycérides mais qu'elle n'est pas négligeable et présente un maximum au $I_{5}{ }^{\mathrm{e}}$ jour. Le taux des acides palmitique et stéarique est, de ce fait, diminué. On trouve aussi, par rapport aux animaux témoins et surtout à partir du $30^{\circ}$ jour d'expérience un pourcentage plus élevé des acides polyinsaturés en $\omega_{3}$ (particulièrement le $\mathrm{C}_{22}: \omega_{5}$ et le $\mathrm{C}_{22}:{ }_{8} \omega_{3}$ ) mais ce phénomène est également observé dans le cas de l'huile de canbra et s'explique par l'apport exogène continu d'acide linolénique par ces 2 huiles. On constate parallèlement à ce résultat et toujours dans le cas des 2 huiles de colza et de canbra, une chute des acides gras polyinsaturés en $\omega_{6}$ dérivés de l'acide linoléique (surtout les $\mathrm{C}_{22}: 4 \omega_{8}$ etC $_{22}: 5 \omega_{8}$ ). Ce résultat est à rapprocher de ce qui avait été observé au niveau des lipides hépatiques (MARTIN, I972).

Enfin, on peut probablement confirmer ici l'augmentation visible du pourcentage d'acide nervonique dans le lot " colza " mais aussi dans le lot " canbra ". Ce résultat mériterait d'être approfondi.

I a composition en acides gras des phospholipides cardiaques est donc aussi influencée par celle des lipides du régime mais ne l'est pas ou peu par la durée de mise au régime.

\section{6. - Structure des triglycérides cardiaques}

chez les animaux ingérant de l'huile de colza

Les premiers résultats obtenus à partir de triglycérides de myocarde de rats ayant ingéré de 1'huile de colza pendant 7 jours (stade aigu de la stéatose) et 6o jours (retour à une valeur quasi-normale des lipides totaux dans l'organe) permettent de dire que les acides érucique et gadoléique sont, à ces 2 stades de l'expérience, préférentiellement situés sur les positions externes des triglycérides alors que les acides oléique et linoléique qui s'accumulent avec les 2 monoènes en $C_{20}$ et $C_{22}$ sont surtout retrouvés sur la position centrale. Les acides gras saturés $\left(\mathrm{C}_{14}\right.$ à $\left.\mathrm{C}_{18}\right)$ sont préférentiellement estérifiés sur la position 2 au $7^{\mathrm{e}}$ jour d'expérience, plutôt au hasard après 60 jours.

\section{7. - Apparition, nature et évolution des lésions myocardiques}

chez les animaux ingérant les huiles de colza et de canbra (tabl. 6)

a) Cas des animaux ayant ingéré de l'huile de colza.

L'infiltration lipidique est diffuse, atteignant toutes les fibres musculaires, ventriculaires ou auriculaires. Elle est évidente aux $3^{\mathrm{e}}$ et $7^{\mathrm{e}}$ jours, et paraît décroître au $15^{\circ}$ jour pour devenir discutable au $3^{\circ}$ jour. Il n'y a donc pas, chez le Rat, de localisation préférentielle de la surcharge lipidique.

Dès le $I 5^{\mathrm{e}}$ jour, et d'une façon plus nette, au $3^{\circ}{ }^{\mathrm{e}}$ jour, apparaissent dans le cytoplasme de certaines cellules, sans topographie particulière, des vacuoles optiquement vides qui ne paraissent pas correspondre à une infiltration lipidique comme le montre 1'étude histochimique des lipides. 
L'existence de ces vacuoles est d'interprétation délicate. Seule la microscopie électronique permet de présager qu'il s'agit de lésions mitochondriales dégénératives $\left({ }^{1}\right)$.

Dès le $I 5^{\mathbf{e}}$ jour, et s'accentuant ensuite, apparaissent des lésions élémentaires dégénératives des fibres musculaires. Peu fréquentes au début elles reproduisent tous les aspects connus des dégénérescences du muscle cardiaque.

\section{TABLEAU 6}

Apparition, nature et évolution des lésions myocardiques chez les animaux ingérant les huiles de colza et de canbra

\begin{tabular}{|c|c|c|c|c|c|c|c|c|c|c|}
\hline \multirow[b]{3}{*}{$\begin{array}{c}\text { Durée } \\
\text { de l'expérience } \\
\text { (jours) }\end{array}$} & \multicolumn{10}{|c|}{ Huile du Régime } \\
\hline & \multicolumn{5}{|c|}{ Colza } & \multicolumn{5}{|c|}{ Canbra } \\
\hline & 3 & 7 & 15 & 30 & 60 & 3 & 7 & 15 & 30 & 60 \\
\hline $\begin{array}{l}\text { Lipides ......... } \\
\text { Vacuoles non lipi- } \\
\text { diques.......... } \\
\text { Lésions élémen- } \\
\text { taires de fibres } \\
\text { Nécrose } \ldots \ldots \ldots \ldots \\
\text { Fibrose } \ldots \ldots \ldots \ldots \\
\end{array}$ & $\begin{array}{c}++ \\
-\end{array}$ & $\begin{array}{c}++ \\
- \\
= \\
-\end{array}$ & $\begin{array}{l}++ \\
\pm \\
\pm \\
=\end{array}$ & $\begin{array}{c} \pm \\
+(3 / 6)(1) \\
+(3 / 6) \\
+(1 / 6) \\
\quad \pm\end{array}$ & $\begin{array}{c}? \\
+(8 / 15) \\
+(8 / 15) \\
+(3 / 15) \\
+(3 / 15)\end{array}$ & $\begin{array}{l}- \\
- \\
- \\
-\end{array}$ & $\begin{array}{l}- \\
- \\
- \\
-\end{array}$ & $\begin{array}{l}- \\
- \\
- \\
-\end{array}$ & $\begin{array}{l}- \\
- \\
\pm \\
-\end{array}$ & $\begin{array}{c}- \\
- \\
+(6 / 16) \\
+(2 / 16) \\
+(2 / 16)\end{array}$ \\
\hline
\end{tabular}

(1) Les nombres entre parenthèses indiquent la fréquence des lésions observées.

A partir du $30^{\circ}$ jour apparaissent des lésions de nécrose, identiques à celles déjà décrites par nous-mêmes et par ABDELLLATIF et al., notamment : disparition de fibres musculaires dans des zones périvasculaires et en particulier dans les régions souspéricardiques. Ces zones, de taille variable, sont infiltrées par des éléments macrophagiques. Au sein de ces zones, ou à leur contact, on distingue une fibrillogenèse collagène débutante, qui marque le premier stade d'une sclérose. Le ventricule gauche et la paroi postérieure paraissent être plus souvent touchés au début mais cette prédominance reste faible.

b) Cas des animaux ayant ingéré de l'huile de canbra.

On ne trouve pas d'infiltration lipidique mais, comme chez les animaux ayant ingéré de l'huile de colza, on constate :

- des lésions élémentaires des fibres myocardiques,

- des lésions de nécrose,

- une fibrillogenèse débutante.

(1) Ces études sont actuellement effectuées, sur les mêmes animaux, par l'équipe de N. VodovaR (Station Centrale de Nutrition, I. N. R. A, Jouy en Josas). 
Ces lésions sont d'apparition plus tardive et moins fréquente mais leur topographie et leur structure sont identiques à celles observées avec le colza.

La comparaison entre les " lésions colza " et les " lésions canbra " ne peut être raisonnablement faite qu'au niveau de leur fréquence. Une étude, par exemple, du nombre de lésions nécrotiques dans l'un et l'autre lot ne pourrait être faite que sur des coupes en série de chaque coeur ce qui représente un travail très long et pratiquement inutile compte tenu de nos expériences antérieures.

c) Cas des animaux ayant ingéré de l'huile d'arachide.

Ces aspects sont totalement absents chez les animaux ayant reçu de 1'huile d'arachide dans leur régime.

\section{DISCUSSION}

\section{I. - Cas de l'huile de colza}

A l'inverse de ce que l'on a pu constater au niveau du foie (MARTIN, I972), les lipides et l'anatomie du myocarde du Rat sont sensiblement affectés par l'incorporation dans le régime d'huile de colza riche en monoènes à très longue chaîne. Nos résultats développent et confirment sur ces deux points ceux obtenus antérieurement (ABDELLATIF et VleS, I970; HouTSMULLER et al., I972; BEARE-ROGERS et al., I97I ; BEARE-ROGERS et NERA, I972 ; RoCQUELIN et CluZAN, I968; RocoueIIN, I972). L'influence de l'huile de colza sur les lipides cardiaques du Rat est quasi immédiate, prolongée, variable dans le temps et revêt deux aspects : qualitatif et quantitatif.

L'accumulation de triglycérides constatée à très court terme dans le myocarde (HOUTSMULLER et al., I970; BEARE-ROGERS et al., I97I ; ROCQUELIN, I972) est encore nettement marquée jusqu'au $30^{\circ}$ jour, puisque, jusqu'à ce stade au moins,

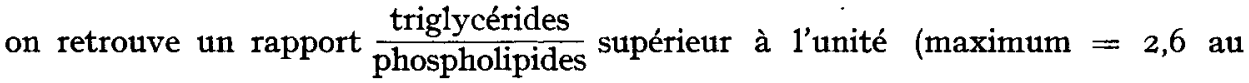
I $5^{\mathrm{e}}$ jour) et par conséquent beaucoup plus élevé que normalement (WITTELs et BRESSLER, I965; WHEELDON et al., I965; Olson et HoEschen, I967; Denton et RANDLE, I967 ; YAMANAKA et OsTWALd, I968; Wood et SNYDER, I968 ; SimoN et Rouser, I969; TURChETTo et al., I969). L'augmentation de la teneur en lipides totaux du myocarde ne passe pas par un maximum bien défini dans le temps. En fait, ce pic est observé, selon les expériences, entre le $3^{\text {e }}$ et 1 e $8^{\text {e jour de mise au }}$ régime, peut être plus tardivement chez certains rats. Les effets de l'huile de colza sur les phospholipides totaux cardiaques (aspect quantitatif) ne sont pas encore clairement définis ici. Néanmoins, nous constatons que la teneur totale de l'organe en acide arachidonique ( $\mathrm{mg} / \mathrm{g}$ de cour) varie peu en fonction de la durée de l'expérience. Or, cet acide gras est surtout présent dans les phospholipides (FrAzER, I962 ; CARrol,L, I965; WAKIL, I970) (voir tabl. 4 et 5) ce qui revient à dire que, quantitativement, ceux-ci sont peu modifiés ce qui irait dans le sens de travaux précédents (BEARE-ROGERS et al., I97I). Une étude récente (BEARE-ROGERS, I972) a néanmoins montré que certaines classes phospholipidiques cardiaques (sphingo- 
myélines) pouvaient être augmentées, à long terme, chez le Rat ingérant de l'huile de navette. C'est là un aspect important et des travaux supplémentaires dans ce sens sont nécessaires.

L'accumulation de triglycérides dans le myocarde est la conséquence d'un blocage de la voie principale d'utilisation des acides gras par cet organe ( $\beta$-oxydation mitochondriale). Cet aspect a déjà fait l'objet de quelques travaux (HouTSMULLER et al., I968; LEMARCHAL et al., I972 ; ChRISTOPHERSEN et BREMER, I972), mais on ne sait pas encore très bien à quel niveau de la chaîne, la réaction est bloquée (extraou intra-mitochondrial). Ce stockage de triglycérides est dû plus spécifiquement à certains acides gras puisque l'on retrouve, selon le moment de l'expérience de 30 à $50 \mathrm{p}$. Ioo d'acides gadoléique et érucique dans les acides gras de cette classe lipidique. Le métabolisme lipidique du myocarde est perturbé par la présence de ces monoènes. Ceux-ci sont, dans les triglycérides cardiaques, préférentiellement estérifiés sur les positions $I$ et 3 du glycérol et, à cet égard, on retrouve une structure qui se rapproche de celle de l'huile de colza. D'autres acides gras insaturés plus courts $\left(\mathrm{C}_{18: 1}\right)$ ou à 2 doubles liaisons $\left(\mathrm{C}_{18: 2}\right)$ sont aussi spécifiquement incorporés dans ces triglycérides cardiaques qui doivent avoir une structure valable pour la cellule (les acides oléique et linoléique sont estérifiés sur la position centrale). Cette dernière explication n'est peut-être pas la seule. Il est en effet intéressant de rappeler que, chez la Gerbille (BEARE-Rogers et NERA, I972), il a été montré que l'huile de navette provoquait à court terme (en une semaine) une accumulation de lipides dans le myocarde comparable quantitativement à celle obtenue chez le Rat dans nos expériences, mais que la teneur en acide érucique de l'organe était, par contre, nettement plus faible (moins de $5 \mathrm{mg} / \mathrm{g}$ de cœur pour une accumulation de lipides totaux de $45 \mathrm{mg} / \mathrm{g}$ ) que chez le Rat. L'acide érucique pourrait donc aussi provoquer une accumulation d'autres acides gras sans pour autant être lui-même largement stocké et ceci, peut-être, en inhibant la $\beta$-oxydation de ces autres acides gras. Les travaux, in vitro, de ChristopHERSEN et BREMER, I972, iraient aussi dans ce sens.

La composition en acides gras des phospholipides cardiaques est aussi modifiée par la présence d'huile de colza dans le régime. C'est important à signaler, car on admet en général (mais ceci a surtout été démontré au niveau du foie) que la nature des acides gras phospholipidiques est peu ou pas modifiée par celle des acides gras exogènes, exception faite évidemment pour les acides gras essentiels. Elle l'est en tout cas beaucoup moins que celle des acides gras des triglycérides. Ici, on constate une incorporation non négligeable des monoènes en $\mathrm{C}_{20}$ et $\mathrm{C}_{22}$ dans les phospholipides qui entraine une diminution des acides gras saturés (palmitique et stéarique) qui sont parmi les chainnes grasses principales des phospholipides (PASCAUD et Strouve, T968). BEARE-Rogers et al. (I972 b) P. C. QUAN et E. LE BRETON (I972) ont également signalé la présence d'acide érucique dans les phospholipides cardiaques du Rat après ingestion pendant quelques jours ( 7 et 3 respectivement) d'un régime riche en acide érucique. Nous retrouvons aussi dans l'organe, une influence de l'acide linolénique apporté par l'huile de colza et l'huile de canbra au niveau du spectre des acides polyinsaturés en $\omega_{3}$ et, par suite, sur celui des acides gras en $\omega_{6}$. Les conséquences de telles modifications de la composition en acides gras des phospholipides sont à étudier.

On ne connaît pas encore très bien les causes de la disparition progressive de la stéatose cardiaque provoquée par l'huile de colza. Nous terminons actuellement 
une étude parallèle à celle présentée ici, sur les lipides du plasma et du tissu adipeux qui devrait apporter des éléments de réponse à ce phénomène d'adaptation apparent.

\section{TABLEAU 7}

Évolution de la teneur en lipides totaux du plasma (mg/g) Évolution des teneurs en acides érucique et gadoléique du plasma des rats ayant ingéré de l'huile de colza (p. Ioo des acides gras totaux)

\begin{tabular}{|c|c|c|c|c|c|}
\hline & \multicolumn{5}{|c|}{ Durée de l'expérience (jours) } \\
\hline & 3 & 7 & 15 & 30 & 60 \\
\hline \multicolumn{6}{|l|}{ Arachide : } \\
\hline Lipides totaux & $\begin{array}{c}\text { 6,8 (3) } \\
(\min .6,0-\text { max. } 7,9)\end{array}$ & $5,1(3)$ & $5,0(3)$ & $4,4(3)$ & $4,3(4)$ \\
\hline \multicolumn{6}{|l|}{ Canbra : } \\
\hline Lipides totaux ...... & $\begin{array}{c}7,5(3) \\
(\min .6,2-\max .9,6)\end{array}$ & $5,4(3)$ & $3,9(4)$ & $4,8(5)$ & $5,0(4)$ \\
\hline \multicolumn{6}{|l|}{ Colza : } \\
\hline Lipides totaux & $\begin{array}{c}8,9(3) \\
(\min .5,6-\max .11,6)\end{array}$ & $4,9(4)$ & $4,5(3)$ & $4,4(3)$ & $3,8(4)$ \\
\hline Acide érucique ....... & 7,4 & 6,1 & 6,6 & 9,9 & 4,8 \\
\hline Acide gadoléique .... & 2,6 & 3,0 & 1,7 & 1,7 & 2,7 \\
\hline
\end{tabular}

Les chiffres entre parenthèses indiquent le nombre de déterminations effectuées.

Nos premiers résultats de ces travaux montrent (tabl. 7) que d'une part, la teneur en lipides totaux du plasma est peu ou pas modifiée par la nature des lipides du régime ou la durée de l'expérience (à l'exception peut-être, au $3^{\mathbf{e}}$ jour et plus particulièrement dans les cas des lipides plasmatiques du lot "colza ", ce qui demande à être confirmé) et, que d'autre part, la teneur en acide érucique du plasma reste relativement constante et peu élevée dans le temps chez les animaux ingérant de 1'huile de colza. Ces données ont été également retrouvées par d'autres auteurs (STRUIJK, I972). Par conséquent, il ne se produit pas, au niveau des lipides sanguins, un phénomène comparable à celui que l'on observe dans le myocarde dès les premières 24 heures d'administration d'huile de colza. L'apport en acide érucique total par le milieu circulant serait donc constant mais son utilisation par le myocarde serait variable dans le temps. On peut préciser ici que la teneur en acide érucique des dépôts gras corporels est également constante dans le temps chez les rats ingérant de 1'huile de colza : 6,7 p. I00, 7,2 p. I00 et 7,I p. Ioo respectivement après 3 , I5 et 60 jours de mise au régime (données non publiées). Il sera évidemment intéressant de préciser les formes de transport de cet acide érucique qui expliqueront mieux son utilisation par le myocarde. Ces résultats partiels permettent néanmoins de supposer que la désinfiltration lipidique du myocarde correspond à une adaptation métabolique de l'organe dans le temps et non à celle d'autres organes comme le foie qui 
ne transforme pas tout l'acide érucique afférent. Notre interprétation sur ce point compléterait celles de Hou'TSMULLER et al. (I970) et de STRUIJK et al. (1973). Ces derniers auteurs viennent en effet de constater que 1'activité de la lipoprotéine lipase (mesurée après injection d'héparine) serait augmentée, chez les rats ayant ingéré de l'huile de colza, pendant la période de désinfiltration lipidique du myocarde.

\section{2. - Cas de l'huile de canbra}

L'huile de canbra ne provoque pas d'accumulation de lipides ni de vacuolisation des cellules du myocarde. Or, comme nous venons de le confirmer ici, cette huile engendre, à terme, des lésions de nécrose comparables à celles obtenues avec l'huile de colza, mais peut-être moins fréquentes et apparaissant plus tardivement : vers le $60^{e}$ jour avec l'huile de canbra, vers le $30^{\ominus}$ avec l'huile de colza. D'autres laboratoires ont, très récemment, confirmé les effets de l'huile de canbra sur le myocarde (PIPY et al., I973).

Il faut remarquer que la fréquence des lésions a été d'interprétation difficile dans notre étude présente du fait de la technique des coupes utilisée qui permettait une meilleure étude de la topographie que de la fréquence. Néanmoins, si nous examinons l'ensemble des résultats obtenus au cours de nos différentes études, nous constatons que la fréquence des lésions provoquées aussi bien par l'huile de colza que par 1'huile de canbra augmente avec le temps et que nous aboutissons à des aspects totalement inhabituels chez des animaux de laboratoire.

L'huile de canbra, comme l'huile de colza, entraine des modifications dans les proportions d'acides gras polyinsaturés en $\omega_{6}$ par rapport aux $\omega_{3}$ et l'apparition d'acide nervonique à l'état de traces dont on peut se demander, dans l'état actuel d'avancement de nos travaux, si elles ont une signification dans la genèse du phénomène pathologique que nous étudions.

En comparant les résultats obtenus avec les huiles de colza et de canbra, on est donc conduit à tirer les conclusions générales suivantes :

Les monoènes à très longue chaîne de 1'huile de colza et l'acide érucique en particulier sont responsables de l'apparition de la stéatose du myocarde et vraisemblablement des lésions anatomiques de cet organe. Il faut rappeler ici que ces deux phénomènes ont en effet été retrouvés en administrant au Rat, non plus de l'huile de colza mais de la triérucine (HoutSMuLLER et al., I970 ; Rocguelin et al., I970; ABDELLATIF et Vles, I970 $b$; ABDELLATIF et Vles, I973).

Malgré tout, on ne peut pas encore conclure définitivement à l'existence d'une corrélation positive entre la stéatose et la lésion. BEARE-ROGERS et NERA (I972 a) ont déjà posé ce problème. Ils ont d'ailleurs aussi montré récemment (BEARERogers et NERA, I972 b) que des lésions cardiaques pouvaient être obtenues à très long terme ( $\mathbf{6}$ semaines) chez le Rat n'ingérant que $5 \mathrm{p}$. Ioo en poids d'huile de navette (à $38 \mathrm{p}$. roo d'acide érucique) sans pour autant mettre en évidence à court terme ( $\mathrm{I}$ semaine) de stéatose cardiaque. Nos résultats vont également dans ce sens : dans le cas de l'huile de colza, les lésions semblent apparaître à distance de l'infiltration lipidique alors que celle-ci a pratiquement disparu; dans le cas de 1'huile de canbra, il y a apparition de lésions sans infiltration lipidique.

Rocouelin et CluZAN (I97I) et ABDEL,LATIF (I972) avaient envisagé la possibilité d'un effet pathologique à long terme sur le myocarde des traces de monoènes 
à très longue chaîne $\left(\mathrm{C}_{20: 1}, \mathrm{C}_{22: 1}\right.$ et $\left.\mathrm{C}_{24: 1}\right)$ encore présents dans l'huile de canbra sans que celles-ci puissent déclencher une stéatose précoce. Cette éventualité avait été retenue, entre autres hypothèses, pour expliquer la seule responsabilité de ces monoènes à longue chaîne dans le double phénomène observé (stéatose et/ou lésion). L'huile de canbra que nous avions testée lors de nos premières expériences contenait en effet $4 \mathrm{p}$. Ioo environ de ces acides gras (dont 2 p. Ioo d'acide érucique) ce qui représentait une ingestion quotidienne d'une centaine de milligrammes par le Rat. Or, l'huile de canbra que nous avons utilisée dans l'expérience présente ne contient guère plus de ces monoènes $(2,8 \mathrm{p}$. Ioo, dont $0,4 \mathrm{p}$. roo d'érucique) que l'huile d'arachide $(2,2 \mathrm{p}$. Ioo dont $0,4 \mathrm{p}$. Ioo d'érucique) qui nous a servi de produit témoin (voir tabl. I) et qui ne provoque pas de lésions myocardiques. Les très faibles quantités d'acide érucique retrouvées dans l'huile d'arachide peuvent s'expliquer soit par l'origine variétale de la graine (BOEKENOOGEN, I968), soit par une légère contamination de l'huile d'arachide, au cours de sa fabrication, par une huile de crucifère (WoLFF, I968). Sans exclure pour autant la possibilité d'une action pathologique à long terme de traces de monoènes à longue chaîne sur le myocarde (mais il faudra expérimentalement en fixer le seuil), on est encore amené, d'après les résultats acquis avec l'huile de canbra, à envisager, comme nous l'avions déjà conclu précédemment (RocQUELIN et Cl,UzaN, r968), la présence d'autres facteurs constitutifs de l'huile susceptibles de provoquer des lésions anatomiques. Venant à l'appui de cette hypothèse, on peut rappeler qu'un certain nombre d'observations histologiques effectuées sur les myocardes des rats ingérant de l'huile de canbra ont révélé quelques caractéristiques des lésions (moment d'apparition, fréquence) légèrement différentes de ce que l'on observe avec l'huile de colza. Dans le cas de cette dernière l'action de facteurs autres que l'acide érucique, s'ils sont aussi présents dans cette huile, serait plus ou moins masquée par les effets spectaculaires et massifs des monoènes à longue chaîne et par conséquent inobservable.

Il est vraisemblable que les études en cours dans de nombreux laboratoires et notamment les recherches effectuées avec l'aide de la microscopie électronique établiront prochainement le type de corrélation existant entre la stéatose et la lésion myocardique. Mais il importe aussi aujourd'hui d'intensifier les recherches sur l'huile de canbra, et d'une manière générale, sur toutes les huiles issues de nouvelles variétés de colza également sélectionnées pour leur absence en acide érucique ( $\left.{ }^{(}\right)$afin de préciser si d'autres éléments constitutifs de ces huiles (naturels ou formés au cours du raffinage) ont des effets pathologiques sur certains organes. Il serait souhaitable et raisonnable que ces études soient achevées avant d'envisager la commercialisation du produit.

Reçu pour publication en avril 1973."

\section{REMERCIEMENTS}

Cette étude a été réalisée avec le concours financier de l'I. N. S. E. R.M. (action thématique programmée $n^{0}$ 6). de Paris.

Les huiles végétales ont été fournies par l'Institut des Corps Gras (I. T. E. R. G.)

(') Ces sélections se poursuivent notamment en France, à l'Institut national de la Recherche agronomique, sous la responsabilité de J. MorICE (Station de Génétique et d'Amélioration des Plantes). 


\title{
SUMMARY
}

\author{
EFFECTS OF RAPESEED AND CANBRA (1) OILS ON CARDIAC L,IPIDS \\ AND ANATOMY OF THE RAT : SHORT-TERM STUDY
}

The authors study the effects of 3 refined vegetable oils (peanut, rapeseed, canbra) on cardiac lipids and anatomy of the rat. The experimental diets contain I5 p. Ioo in weight (30 p. Ioo calories) of lipids The rats are taken at weaning and sacrificed 3, 7, I5, 30 and 60 days after beginning the diet. Rapeseed oil very quickly causes a large accumulation of triglycerides rich in long-chain monoenes (erucic and gadoleic acids) which reaches a maximum during the first week. This abnormal storage of long-chain monoenes in the cardiac triglycerides causes other fatty acids (oleic and linoleic) to be stored and modifies the glyceridic structure. Cardiac steatosis disappears gradually with time, but 60 days after the beginning of the experiment, fair amounts of erucic acid are still found in the organ (8.I p. 100). The hypothesis of metabolic adaptation of the myocardium is suggested. Erucic acid is also found in the cardiac phospholipids (4.8 to I 2 p. Ioo) on day 3 of the diet. None of these phenomena are found with peanut and canbra oils.

Rapeseed and canbra oils, contributing linolenic acid, change the myocardic spectrum of polyunsaturated acids, $C_{20}$ and $C_{22}$ in $\omega_{6}$ and $\omega_{3}$.

Myocardic lesions are again found in rats ingesting both rapeseed and canbra oils. These results pose the problem again of a cause and effect relationship which may exist between steatosis and cardiac lesion and that of erucic acid being solely responsible for these two pathological effects.

(1) Canbra $=$ Contraction of Canadian brassica Name given by Canadian geneticists to rapeseed oil poor in erucic acid.

\section{RÉFÉRENCES BIBLIOGRAPHIQUES}

Abdellatif A. M. M., I972. Cardiopathogenic effects of dietary rapeseed oil. Nutr. Rev., 30, 2-6.

Abdellatif A. M. M., Vles R. O., 1970 a. Physiopathological effects of rapeseed oil and canbra oil in rats. Proc. internat. conf. science, technology and marketing of rapeseed and rapeseed products. Sainte-Adèle (Canada), 423-434.

Abdellatif A. M. M., Vles R. O., I970 $b$. Pathological effects of dietary rapeseed oil in rats. Nutr. Metabol., 12, 285-295.

AbDellatif A. M. M., Vles R. O., 1971. Long-term pathological effects of dietary rapeseed oil in rats and rabbits. Voeding, 32, 602-6II.

Abdellatif A. M. M., Vles R. O.,x973. Short and long term pathological effects of glyceryl trierucate and of increasing levels of dietary rapeseed oil in rats. Nutr. Metabol., 15,219-23I.

Beare-Rogers J. L., Nera E. A., Heggtveit H. A., r971. Cardiac lipid changes in rats fed oils containing long chair: fatty acids. Can. Inst. Food Technol. J., 4, 120-124.

Beare-Rogers J. L., Nera E. A., I972 a. Cardiac fatty acids and histopathology of rats, pigs, monkeys and gerbils fed rapeseed oil. Comp. Biochem. Physiol. B., 41, 793-80o.

Beare-Rogers J. L., Nera E. A., I972 b. Cardiac lipids in rats and gerbils fed oils containing $\mathrm{C}_{22}$ fatty acids. Lipids, 7, 548-522.

BeARE-Rogers J. L., I972. Nutritional effects of docosenoic acid. II Congr. Mond. Matieres grasses de Göteborg (Suède). Abstracts, 43.

BodaK A., I972. Etude au microscope électronique des lesions cellulaires myocardiques induites par une surcharge en huile de colza chez le Rat. Thèse Médecine, Paris.

BoE KE NOOGEN H. A., 1968. Analysis and characterization of oils, fats and fat products. 2, p. 288. Interscience Publishers.

Brown J. L., Johnston J. M., I962. Radioassay of lipid components separated by thin-layer chromatography. J. Lipid Res., 4, 480-481. 
Carroll K. K., I965 Dietary fat and the fatty acid composition of tissue lipids J. Am. Oil Chem. Soc., 42, $516-529$.

Christophersen B. O., Bremer J., rg72. Inhibitory effect of erucylcarnitine on the oxidation of palmitate by Rat heart mitochondria. F.E. B.S. Letters, 28, 230-23I.

Denton R. M., Randee P. J., 1967. Concentrations of glycerides and phospholipids in Rat heart and gastrocnemius muscles. Effects of alloxan diabetes and perfusion. Biochem. J., 104, 416-422.

Folch J., Lees M., Slonne Stanley G. H., i957. A simple method for the isolation and purification of total lipids from animal tissues. J. Biol. Chem., 226, 497-509.

Frazer A. C., I962. Fate of dietary fat in the body. Nature, 194, 908-909.

Houtsmuller U. M. T., Struijk C. B., Ven Der Beek A., I97o. Decrease in rate of ATP synthesis of isolated rat heart mitochondria induced by dietary erucic acid. Biochim. Biophys. Acta., 218, 564566.

Houtsmuller U. M. T., Struijk C. B., Van Der Beek A., r972. Biochemical effects of dietary very long chain fatty acids on rat heart and liver. (Abstr.) J. Am. Oil Chem. Soc., 49, $304 \mathrm{~A}$.

Lemarchal P., Clouet P., Blond J. P., r972. $\beta$-oxydations comparées des acides érucique et oléque par les mitochondries de cour de Rat in vitro. C. R. Acad. Sc. Paris, 274, 196r-1963.

Luddy F. E., Barford R. A., Herb S. F., Magidman P., Riemenschneider R. W., I964. Pancreatic lipase hydrolysis of triglycerides by a semi-micro technique. J. Am. Oil Chem. Soc., 41 , $693-696$.

MaRTIN B., I972. Influence des huiles de colza et de canbra et de mélanges de triglycerides homogènes reproduisant certaines caractéristiques de ces huiles sur les lipides hépatiques du Rat. Thèse $3^{\circ}$ Cycle, Dijon.

Mattson F. H., Volpenheim R. A., r96I. The specific distribution of fatty acids in the glycerides of vegetable oils. J. Biol. Chem., 236, 189r-1894.

Munsch N., I97o. Influence de l'ingestion de triélaïdine sur la composition en acides gras des lipides hépatiques et plasmatiques de Rat. Ann. Nutr. Alim., 24, 24-40.

Olson R. E., Hoeschen R. J., I967. Utilisation of endogenous lipid by the isolated perfused rat heart. Biochem. J., 103, 796-80r.

Pascaud M., Strouve C., r968. Devenir métabolique des acides ${ }^{14} \mathrm{C}$-linoléique et palmitique chez le Rat en croissance. I. Oxydation et utilisation énergétique. Bull. Soc. Chim. Biol., 50, 569-577.

Pipy B., Gaillard D., Derache R., r972. Influence de régimes contenant les huiles de colza et de canbra sur le système réticulo-endothélial du Rat. J. Physiol. Paris, 64, 439-456.

Quan P. C., Le Breton E., I972. Répartition et composition en acides gras des classes de phospholipides dans le myocarde du Rat ayant ingéré, durant 3 jours, un régime contenant de l'huile d'arachide, de canbra ou de colza. Influence du jeâne et des lipotropes. $C$. $R$. Acad. Sc. Paris, 275, I27Ir274.

Rocouelin G., Cluzan R., rg68. L'huile de colza riche en acide érucique et l'huile de colza sans acide érucique : valeur nutritionnelle et effets physiologiques chez le Rat. I. Effets sur la croissance, l'efficacité alimentaire et l'état de différents organes. Ann. Biol, anim. Bioch. Biophys., 8, 395-406.

Rocouelin G., Martin B., Cluzan R., r97o. Comparative physiological effects of rapeaseed and canbra oils in the Rat : influence of the ratio of saturated to monounsaturated fatty acids. Proc. internat. conf. science, technology and marketing of rapeseed and rapeseed products. Sainte-Adele (Canada). 405-422.

Rocouelin G., Cluzan R., r971. L'huile de colza : ses effets particuliers au niveau du myocarde. Cah. Nut. Diet., 6, 85-95.

Rocouelin G., I972. L'huile de colza et l'huile de canbra : effets à très court terme sur les lipides cardiaques et hépatiques du Rat sevré. C. R. Acad. Sc. Paris, 274, 592-595.

Simon G., Rouser G., I969. Species variations in phospholipid class distribution of organs. II. Heart and skeletal muscle. Lipids , 4, 607-614.

STRUijk C. B., I972. The effect of dietary erucic acid on rat heart and liver. (Abstr.). 15th Internat. Conf. Biochemistry of Lipids. I. C. B. L. The Hague (Netherlands). A 3 I.

StruijK C. B., Houtsmulier U. M. T., JANSEN H., HülsmanN W. C., I973. Increase in post heparin lipoprotein lipase activity in rat plasma by dietary erucic acid. Biochim. Biophys. Acta, 296, $253-256$.

Turchetro E., Weiss H., Bocchini G., Formigarini E., I969. Die Gewebslipoide der Ratte in verschiedenen Altersstufen. Nutr. Dieta, 11, rog-1 14.

Vies R. O., Abdellatif A. M. M., 1970. Long-term effects of rapeseed oil in rats and rabbits. (Abstr.). J. Am. Oil Chem. Soc., 47, 295-356 A.

- Vodovar N., Desnoyers F., Cluzan R., Marson A. M., Levillain R., 1972. Accumulation de lipides dans le myocarde de rats ayant reçu de l'huile de colza dans leur régime. Aspect ultrastructural. C. R. Acad. Sc. Paris, 274, 3109-3II2.

Wakil S. J., r97o. Lipid Metabolism. Academic Press, Inc.

Walker B. L., I972. Deposition of erucic acid in Rat tissue lipids. Nutr. Metabol., 14, 8-I6.

Walker B. L., Atkinson S. M., Zehaluk C. M., Mackey M. G., I972. Comparative deposition of erucic acid in rat adrenal and ovarian lipids. Comp. Biochem. Physiol. B., 42, 6I9-625. 
WheEldoN L. W., Schumert Z., TURNer D. A., r965. Lipid composition of heart muscle homogenate. J. Lipid Res., 6, $48 \mathrm{I}-497$.

WitTels B., Brisster R., 1965. Lipid metabolism in the newborn heart. J. Clin. Invest., 44, 16391646.

Wol.rF J. P., x968. Manuel d'analyse des corps gras, p. 335. Azoulay, éditeur.

WOOD R., SNYDER F., r968. Quantitative determination of alk-r-enyl and alkyl-glyceryl ethers in neutral lipids and phospholipids. Lipids, 3, r29-135.

$\rightarrow$ YamanaKa W., Ostwald R., Ig68. Lipid composition of heart kidney and lung in guimea pigs made anemic by dietary cholesterol. J. Nutrit., $85,38 \mathrm{r}-387$. 